\title{
A novel degradation state indicator for steam generators of nuclear power plants
}

\author{
Luca Pinciroli \\ Energy Department, Politecnico di Milano, Via Lambruschini 4, 20156, Milan, Italy. \\ E-mail: luca.pinciroli@polimi.it
}

Piero Baraldi

Energy Department, Politecnico di Milano, Via Lambruschini 4, 20156, Milan, Italy. E-mail:piero.baraldi@polimi.it

\begin{abstract}
Ahmed Shokry
Energy Department, Politecnico di Milano, Via Lambruschini 4, 20156, Milan, Italy. Center for Applied Mathematics, Ecole Polytechnique, Route de Saclay, 91120 Palaiseau, France.

E-mail:ahmed.shokry@polimi.it

Enrico Zio

Energy Department, Politecnico di Milano, Via Lambruschini 4, 20156, Milan, Italy. MINES ParisTech, PSL Research University, CRC, Sophia Antipolis, France.

Eminent Scholar, Department of Nuclear Engineering, College of Engineering, Kyung Hee University, Republic of Korea.E-mail: enrico.zio@polimi.it
\end{abstract}

We develop a degradation indicator for nuclear power plants steam generators, based on the use of signal measurements collected by sensors during plant operational transients between two successive maintenance interventions. Given the unavailability of information about the real degradation state during operation, an unsupervised approach is adopted. It consists in the extraction of several features from raw signals and in the selection of those features which best describe the degradation state evolution within a multi-objective optimization framework. The two considered objectives are the monotonicity and trendability of the features.

Keywords: Condition-based maintenance, Degradation assessment, Unsupervised, Feature selection, Nuclear power plant, Steam generator.

\section{Introduction}

One of the main degradation mechanisms of Steam Generators (SG) of Nuclear Power Plants (NPP) is the Tube Support Plates (TSP) clogging (Corredera, Alves-Vieira and de Bouvier, 2008), which consists in the deposition of particles on the quatrefoil holes of the TSP. The consequent reduction of the flow area leads to the formation of high velocity zones that enhance tube vibrations, which may induce tube cracking. This causes a reduction of the outlet steam pressure and, consequently, of the NPP overall power production.

Mechanical and chemical cleaning are maintenance interventions periodically performed to reduce or remove the SG deposits (Riznic, 2017). Both of them are long and expensive operations, which cause the unavailability of the SG for several hours, can damage some of the SG parts (Girard, 2014) and produce large volumes of wastes.
Given the criticality of SGs and the large costs associated to their maintenance, the adoption of Condition-Based Maintenance (CBM) policies based on the estimation of the SG degradation is crucial (Di Maio, Antonello and Zio, 2018). The key quantity in $\mathrm{CBM}$ is the component degradation state, which can be directly measured or indirectly computed from other measured signals.

Different types of Non-Destructive Tests (NDTs), such as televisual and eddy current inspections, have been developed to assess SG degradation level. Both techniques have practical limitations: televisual inspection allows assessing only the degradation of the upper part of the SG, whereas eddy current inspection can be applied only if the blockage levels are lower than 50\% (Corredera, Alves-Vieira and de Bouvier, 2008).

In this work, we propose a novel three-step method for the identification of the degradation state of the TSP of SGs on the basis of the evolution of monitored signals during plant

Proceedings of the 30th European Safety and Reliability Conference and the 15th Probabilistic Safety Assessment and Management Conference Edited by Piero Baraldi, Francesco Di Maio and Enrico Zio Copyright (C) ESREL2020-PSAM15 Organizers.Published by Research Publishing, Singapore. ISBN/DOI: 978-981-14-8593-0 
operational transients. In the first step, features are extracted from the monitored signals collected during operational transients. In the second step, the goodness of the extracted features is systematically evaluated with respect to the two metrics of monotonicity and trendability that represent preferable properties of degradation indicators (Coble and Hines, 2009). Finally, in the third step, the problem of selecting the features for the construction of the degradation indicator is framed as a multi-objective optimization problem and the selected features are used to build the degradation indicator.

The performance of the proposed method is shown using real data collected during 25 years of operation of a fleet of NPP SGs.

\section{Case Study}

We consider $Z=81$ SGs of a 900 MW NPP fleet. The available dataset contains the values of $\mathrm{K}=$ 15 signals describing the SGs behavior during tests periodically performed during 17 years of operation, which are characterized by a decreasing power ramp from $100 \%$ to $40 \%$.

During each transient, the signals are acquired for a $2 \mathrm{~h}$ period at a sampling frequency of $0.5 \mathrm{~Hz}$. The dataset contains a total number of $N_{\text {tot }}=$ 2694 operational transients performed by the different SGs. Although the considered power transients usually last for around 2 hours, only the decreasing power ramp is a well standardized and repeatable process, whereas the signal evolution in the remaining part of the transient remarkably depends on the operational conditions and on the demand at the grid side. For this reason, we consider the evolution of the signals during a period of approximately $1200 \mathrm{~s}$.

We indicate with $N_{z}$ the number of transients collected from the generic $z$-th element of the fleet and with $N_{t o t}=\sum_{Z=1}^{Z} N_{Z}$ the total number of transients collected from the entire fleet. The value of the $k$-th signal measured during the $n_{z}$-th transient of the $z$-th element at time $t$ is indicated by:

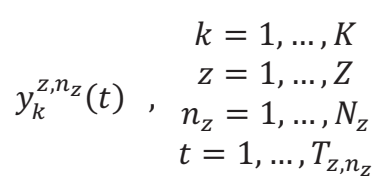

where $T_{z, n_{z}}$ represents the duration of the $n_{z}$-th transient of the $z$-th element of the fleet.

The objective is to identify the state of degradation $d_{z_{\text {test }}}^{E}\left(n_{z_{\text {test }}}\right)$ of a test element (SG) using the signal measurements
$y_{k}^{z_{\text {test }}, n_{z} \text { test }}(t), k=1, \ldots, K$ collected during an operational transient.

\section{Method}

The degradation indicator is developed based on the extraction of a large set of features from the monitoring signals, the evaluation of the monotonicity and trendability of the extracted features and the construction of the degradation indicator from the selected features.

\subsection{Feature Extraction}

The feature extraction phase aims at extracting $J$ different features, $x_{j, k}^{z}\left(n_{z}\right), j=1, \ldots J$, from the evolution of the $k$-th signal $y_{k}^{z, n_{z}}(t)$, during the generic transient $n_{z}$ of element $z$. Notice that the quantity $x_{j, k}^{z}\left(n_{z}\right)$ is explicitly written as a function of the transient $n_{z}$ to indicate that it describes the element state at the time in which the $n_{z}$-th transient occurs.

Different types of features have been considered: statistical features (e.g. mean, skewness), time-frequency features (e.g. wavelet, energy) (Sharp, 2012) and ad-hoc features (e.g. coefficients of interpolating parabola).

\subsection{Features goodness evaluation}

According to (Coble and Hines, 2009; Baraldi, Bonfanti and Zio, 2018), a good degradation indicator is characterized by two main desirable properties:

\subsubsection{Monotonicity}

Since equipment typically does not exhibit a selfhealing behavior, degradation can only increase as time passes. Therefore, a good degradation indicator is expected to progressively and monotonically increase or decrease with time. To assess how much the feature $x_{j, k}^{Z}(\cdot)$ shows a monotonic trend, two metrics are considered in this work. The first one is based on the MannKendall test (Mann, 1945), whereas the second one is taken from (Coble and Hines, 2009).

The Mann-Kendall test formulates two hypotheses (Pohlert, 2015):

- The null hypothesis, $H_{0}$, is that the trend of the feature $x_{j, k}^{Z}(\cdot)$ is not monotonic;

- The alternative hypothesis, $H_{A}$, is that the trend of the feature $x_{j, k}^{Z}(\cdot)$ is monotonic. 
Considering the $j$-th feature extracted from the $k$-th signal during the life of the $z$-th element along all the transients (from the first $n_{z}=1$ to the last $\left.n_{z}=N_{z}\right), x_{j, k}^{z}\left(1: N_{z}\right)$, the Mann-Kendall test provides a binary outcome, $M K_{j, k, z}=(0,1)$, with 1 indicating that the feature $x_{j, k}^{z}$ has a monotonic trend and 0 otherwise. The global Mann-Kendall monotonicity index, M.I. MK ${ }_{\cdot j, k}$, associated to the generic $j$-th feature extracted from the $k$-th signal, $x_{j, k}$, is defined as the average value of the outcome, $M K_{j, k, z}$, of the Mann-Kendall test applied to all the $Z$ elements of the fleet:

$$
M . I_{\cdot j, k}^{M K}=\frac{\sum_{z=1}^{Z} M K(j, k, z)}{Z}
$$

Since $M K_{j, k, z}$ can only assume values 0 or 1 , a limitation of $M . I_{\cdot j, k}^{M K}$ is that it can assume only the discretized values, $0, \frac{1}{Z}, \frac{2}{Z}, \ldots, 1$, which can create a difficulty in the ranking of the features when handling data collected from a small number of similar fleet elements, $Z$. For this reason, another monotonicity index M.I. ${ }^{\text {der }}$, based on the study of the feature discrete derivatives proposed in (Coble and Hines, 2009) is also considered. The derivative-based monotonicity index is defined by:

$$
\begin{aligned}
& M . I_{\cdot j, k, Z}^{\text {der }}=\left|\frac{(\# \Delta>0)-(\# \Delta \leq 0)}{N_{Z}-1}\right| \\
& M . I_{\cdot j, k}^{\text {der }}=\operatorname{mean}_{Z}\left(M \cdot I_{\cdot j, k, Z}\right)
\end{aligned}
$$

where $\# \Delta>0$ and $\# \Delta \leq 0$ indicate the number of times the discrete difference $x_{j, k}^{z}\left(n_{z}+\right.$ 1) $-x_{j, k}^{z}\left(n_{z}\right)$ with $n_{z}=1, \ldots, N_{z}-1$, is positive and negative, respectively, and the denominator in Eq.(3) counts the number of times the discrete difference is computed. The M.I. der monotonicity index provides values within the range $[0,1]$. Also in this case, M.I. $._{j, k}^{d e r}=0$ indicates a completely non-monotonic behaviour, whereas the value of 1 indicates an ideal or completely monotonic behaviour.

\subsubsection{Trendability}

A good degradation indicator is expected to show a similar functional evolution (i.e. "trend") during the lives of similar elements of the same fleet. In order to measure the trendability of the $j$-th feature extracted from the $k$-th signal collected from the $z$-th elements, $x_{j, k}^{Z}\left(1: N_{z}\right)$, the trendability index suggested by Cobles and Hines (2009) is here considered:

$$
\begin{aligned}
& T R_{j, k, Z}=\frac{\# \Delta>0}{N_{z}-1}+\frac{\# \Delta^{2}>0}{N_{z}-2} \\
& T . I_{\cdot j, k}=1-\operatorname{std}\left(T R_{j, k, Z}\right)
\end{aligned}
$$

where $\# \Delta^{2}=x_{j, k}^{z}\left(n_{z}+1\right)-2 x_{j, k}^{z}\left(n_{z}\right)+$ $x_{j, k}^{Z}\left(n_{z}-1\right)$, with $n_{z}=2, \ldots, N_{z}-1$, is the number of second-order differences. Notice that $T R_{j, k, z}$ is a relative measure of the number of the evaluated positive first, $\Delta$, (second, $\Delta^{2}$ ) discrete derivatives with respect to the number of the total evaluations of first (second) discrete derivatives of the $j$-th feature extracted from the $k$-th signal collected from the $z$-th element, whereas, $s t d_{Z}$ represents the standard deviation evaluated with respect to the $Z$ similar elements of the fleet. This metric can assume any value in the range $[0,1]$, where 0 identifies the absence of trendability for the $j$-th feature and 1 represents a perfectly trendable behavior.

\subsection{Features selection}

The definition of degradation indicators requires the identification of the features with the most satisfactory trade-offs between monotonocity and trendability. The vector of objective functions $f=\left(f_{1}, f_{2}, f_{3}\right)$ defined by the three indexes (M.I.j. $\left.{ }_{\cdot j . k}^{M K}, M . I_{\cdot j, k}^{d e r}, T \cdot I_{\cdot j, k}\right)$ is computed for each one of the $J \cdot K$ extracted features, $x_{j, k}$, and the Pareto optimal set of features is identified (Baumgartner, Magele and Renhart, 2004). To reduce the number of the candidate optimal features and eliminate those with unsatisfactory trade-off among the different objectives, we identify a subset of the Pareto front by assigning a relative importance to the different objectives. In particular, we associate to each objective function, $f_{i}$, a weighted utility function (Branke, Kaußler and Schmeck, 2001; Baraldi, Pedroni and Zio, 2009) defined by:

$$
\Omega_{i}(f)=f_{i}+\sum_{i=1, j \neq i}^{3} a_{i j} \cdot f_{j}, i, j \in(1,2,3)
$$

where $a_{i j}$ quantifies the amount of gain in the $j$-th objective required to accept a loss of one unit in the $i$-th objective and $a_{i i}$ is by definition equal to 1. The dominance is, then, evaluated with respect to the vectors of objective functions $\Omega=$ 
$\left(\Omega_{1}, \Omega_{2}, \ldots, \Omega_{F}\right)$ with $F=3$. The definition of $\Omega$ requires the setting of $F^{2}-F$ trade-offs on the basis of the relative importance that the analyst gives to each objective. In this work, since similar feature trends have been observed in different SGs of the same plant due to the fact that they experience the same operational conditions, we have concluded that features with large trendability indexes are not necessarily strong degradation indicators. For this reason, the weights associated to $T \cdot I_{\cdot j, k}$ are set lower than the ones of M.I. $I_{. j . k}^{M K}$ and M.I..$_{\cdot j, k}^{d e r}$. In Table 1 , the values of the weights associated to $M . I_{\cdot j \cdot k}^{M K}$, $M . I_{\cdot j, k}^{d e r}$ and $T . I_{\cdot j, k}$ are reported.

Table 1 . Weighted utility function.

\begin{tabular}{cccc}
\cline { 2 - 4 } & $M . I_{\cdot j, k}^{M K}$ & $M . I_{\cdot j, k}^{\text {der }}$ & T.I. $_{\cdot j, k}$ \\
\hline$\Omega_{M \cdot I_{\cdot j, k}^{M K}}^{M K}$ & 1 & 0.1 & 0.05 \\
\hline$\Omega_{M . I . j, k}^{\text {der }}$ & 0.1 & 1 & 0.05 \\
\hline$\Omega_{T \cdot I \cdot I_{j, k}}$ & 0.1 & 0.1 & 1 \\
\hline
\end{tabular}

\section{Validation of the Degradation Indicator}

The validation of the proposed methodology requires the evaluation of the accuracy of the selected degradation indicator with respect to the results of some NDTs. Since the different features have different units of measurement and ranges, they are first normalized on the same scale, which is taken equal to that of the NDTs. In practice, the normalized value, $d_{z}^{j, k}\left(n_{z}\right)$, of the optimal feature $j$ extracted from signal $k$ of element $z$ at time $n_{z}$ is defined by:

$$
d_{z}^{j, k}\left(n_{Z}\right)=\alpha_{j, k}^{Z} x_{j, k}^{Z}\left(n_{Z}\right)+\beta_{j, k}^{Z}
$$

where the parameters $\alpha_{j, k}^{Z}, \beta_{j, k}^{z}$ of the linear relationship are independently set for each feature $j$ extracted from signal $k$ of element $z$ using the Ordinary Least Squares (OLS) method. The objective of the setting is to minimize the error between the first two NDT results $D_{z}(h)$ and the corresponding normalized feature values $d_{z}^{j, k}\left(n_{z}^{h}\right)$, where $n_{z}^{h}$ is the time of the operational transient closest to the time of the $h$-th NDT, with $h=1,2$. The normalized selected features are, then, aggregated into a single, robust degradation indicator, $d_{z}^{E}\left(n_{z}\right)$, with $n_{z}=1, \ldots, N_{z}$, by considering their median:
$d_{z}^{E}\left(n_{z}\right)=\operatorname{median}_{\{j, k \in \text { optimal pareto set }\}} d_{z}^{j, k}\left(n_{Z}\right)$

The median operator has been chosen since it has been shown to be more robust to possible outliers than other operators such as the mean (Leys et al., 2013).

To assess the performance of the degradation indicator, we introduce an accuracy metric which considers the absolute error between the degradation state estimation, $d_{z}^{E}\left(n_{z}^{q}\right)$ and the corresponding NDT measurement $D_{z}(q)$ :

$$
e_{z}(q)=\left|D_{z}(q)-d_{z}^{E}\left(n_{z}^{q}\right)\right|
$$

with $q=3, \ldots, Q_{z}$, where $Q_{z}$ is the number of NDTs available for the $z$-th element Notice that the first two NDT measurements, $q=1,2$, are not considered since they have been already used for the data normalization. The mean error and the error standard deviation of the degradation indicator in the estimation of the degradation state of the generic $q$-th NDT test are, respectively:

$$
\begin{gathered}
E_{q}=\frac{\sum_{z=1}^{Z_{q}} e_{z}(q)}{z_{q}} \\
\sigma_{q}=\sqrt{\frac{\sum_{z=1}^{Z_{q}}\left(e_{z}(q)-E_{q}\right)^{2}}{z_{q}}}
\end{gathered}
$$

where $Z_{q}$ indicates the number of test elements that have performed at least $q$ NDTs. Then, the overall mean estimation error is:

$$
\bar{E}=\frac{\sum_{q}\left(Z_{q} E_{q}\right)}{\sum_{q} Z_{q}}
$$

\section{Results}

A total of $\mathbf{J}=93$ features have been extracted from each one of the $\mathrm{K}=15$ signals of interest. The obtained Pareto optimal set is formed by 5 features, extracted from three different signals (Figure 1- $(b, c, d, e, f)$ ) and the degradation indicator is obtained through their aggregation (Eq. (9)). The results have been verified to be robust with respect to small perturbations of the entries of the weighted utility function.

Figure 1-(a) shows an example of the evolution of the degradation level estimate for a steam generator. Notice:

- the good accuracy with respect to the available NDT data (green crosses)

- the monotonic trend. The two points in which the degradation level shows a sudden decrease (at the dashed and 
dashed-dotted vertical lines) correspond to maintenance interventions performed on the considered SG. In particular, the largest jump is associated to chemical cleaning, whereas the smallest one is associated to mechanical cleaning.

Notice that the first two features (Figure $1 b, c$ ), which are characterized by high trendability and low monotonicity, and, therefore, provide a not satisfactory estimation of the component degradation, have low impact on the final degradation assessment thanks to the use of the median operator.

Table 2 reports the average performance of the degradation indicator in terms of mean error, $E_{q}$, and corresponding standard deviation, $\sigma_{q}$, in the estimation of the degradation state in correspondence of the $q$-th NDT performed on the components. It can be noticed that the majority of the errors is smaller than $10 \%$, which is comparable with the error of the ETVs, especially when the degradation state is low. The total mean estimation error, $\bar{E}$, is 7.6.
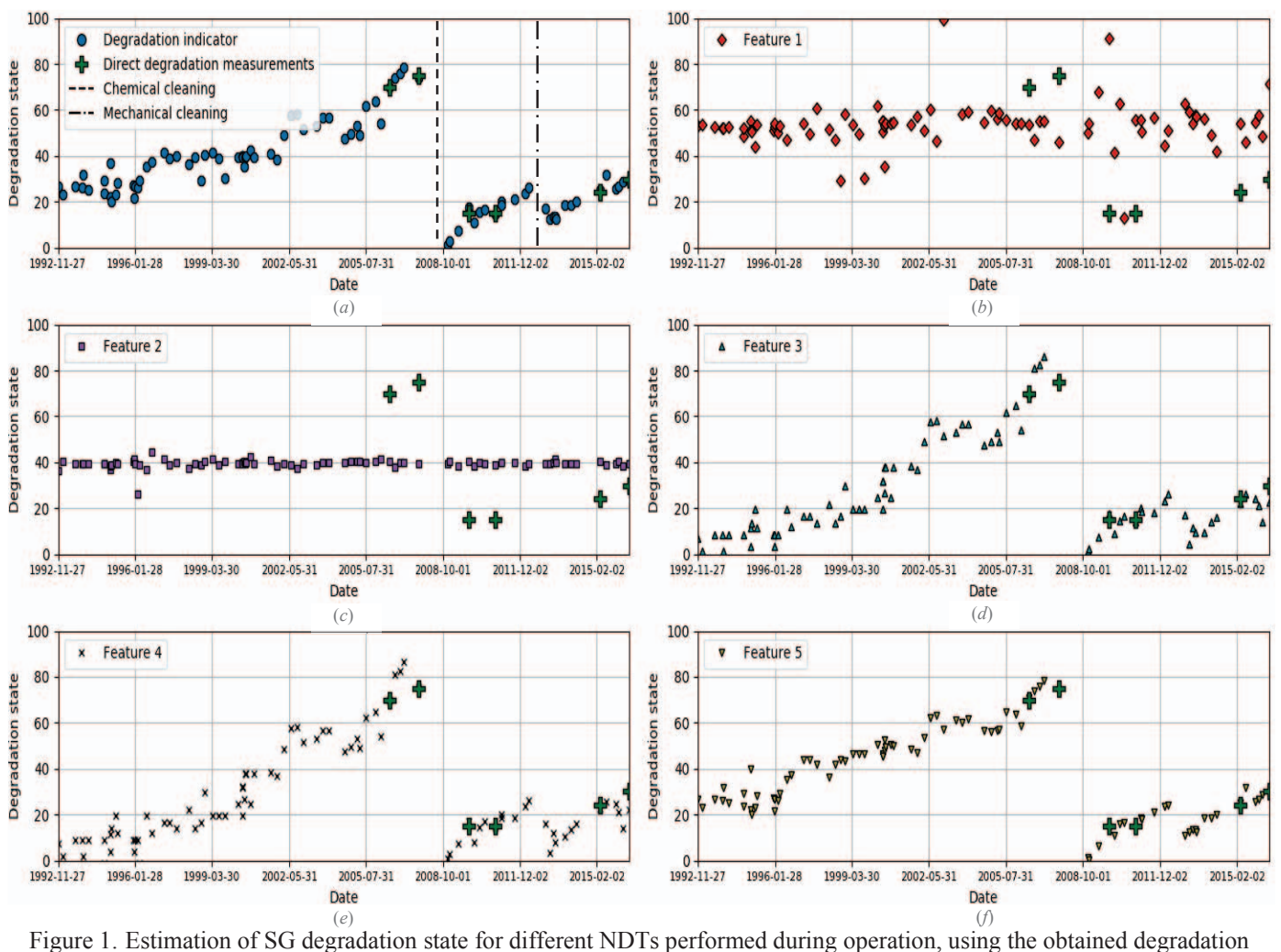

Figure 1. Estimation of SG degradation state for different NDTs performed during operation, using the obtained degradation indicator $(a)$ and the five individual features $(b, c, d, e, f)$.

Table 2. Performance of the proposed degradation indicator applied to the $900 \mathrm{MW}$ fleet data in terms of estimation error $[\%]$.

\begin{tabular}{clllll}
\hline $\boldsymbol{q}$ & $\mathbf{3}$ & $\mathbf{4}$ & $\mathbf{5}$ & $\mathbf{6}$ & $\mathbf{7}$ \\
\hline$E_{q}$ & 14.9 & 6.5 & 6.0 & 6.8 & 12.4 \\
\hline$\sigma_{q}$ & 7.9 & 0.6 & 3.9 & 5.0 & 3.3 \\
\hline
\end{tabular}

\section{Conclusions}

This work proposes a novel unsupervised method for the definition of a degradation indicator for nuclear power plant steam generators. It allows exploiting the information contained in the large amounts of monitoring signal data collected by the plant supervision system. The method is based on three steps: $i$ ) features extraction, $i i$ ) evaluation of the goodness of the extracted features with respect to the monotonicity and trendability and iii) multi-objective selection of the optimal features and their aggregation to define the degradation indicator. The proposed approach has been shown able to identify the steam generator degradation state from signal measurements acquired during plant operational transients with errors comparable to those obtained performing ad-hoc expensive non-destructive tests. 


\section{Acknowledgements}

We acknowledge Redouane Seraoui and Carole Mai (Électricité de France R\&D, 6 quai Watier, Chatou, France), for providing the case study and the dataset.

\section{References}

Ayodeji, A. and Liu, Y. kuo (2019) 'PWR heat exchanger tube defects: Trends, signatures and diagnostic techniques', Progress in Nuclear Energy. doi: 10.1016/j.pnucene.2018.12.017.

Baraldi, P., Bonfanti, G. and Zio, E. (2018) 'Differential evolution-based multi-objective optimization for the definition of a health indicator for fault diagnostics and prognostics', Mechanical Systems and Signal Processing, 102, pp. 382-400.

Baraldi, P., Pedroni, N. and Zio, E. (2009) Optimal power system generation scheduling by multi-objective genetic algorithms with preferences, Reliability Engineering and System Safety. doi: 10.1016/j.ress.2008.04.004.

Baumgartner, U., Magele, C. and Renhart, W. (2004) 'Pareto Optimality and Particle Swarm Optimization', IEEE Transactions on Magnetics, 40(2), pp. 1172-1175.

Branke, J., Kaußler, T. and Schmeck, H. (2001) 'Guidance in evolutionary multi-objective optimization', Advances in Engineering Software, 32(6), pp. 499-507. doi: 10.1016/S0965-9978(00)00110-1.

Coble, J. and Hines, J. W. (2009) 'Identifying optimal prognostic parameters from data: a genetic algorithms approach', Proceedings of the Annual Conference of the Prognostics and Health Management Society, pp. 1-11. Available at: https://www.phmsociety.org/sites/phmsociety .org/files/phm_submission/2009/phmc_09_6 9.pdf.

Corredera, G., Alves-Vieira, M. and de Bouvier, O. (2008) 'Fouling and TSP Blockage of Steam Generators on EDF Fleet: Identified Correlations with Secondary Water Chemistry and planned Remedies', in Water chemistry of nuclear reactor systems.

Girard, S. (2014) Physical and Statistical Models for Steam Generator Clogging Diagnosis.
Springer International Publishing. doi: 10.1007/978-3-319-09321-5.

Leys, C. et al. (2013) 'Detecting outliers: Do not use standard deviation around the mean, use absolute deviation around the median', Journal of Experimental Social Psychology. Elsevier Inc., 49(4), pp. 764-766. doi: 10.1016/j.jesp.2013.03.013.

Di Maio, F., Antonello, F. and Zio, E. (2018) 'Condition-based probabilistic safety assessment of a spontaneous steam generator tube rupture accident scenario', Nuclear Engineering and Design. doi: 10.1016/j.nucengdes.2017.10.020.

Mann, H. B. (1945) 'Nonparametric Tests Against Trend', Econometrica, 13(3), pp. 245-259.

Pohlert, T. (2015) 'Non-Parametric Trend Tests and Change-Point Detection', CC BY-ND 4.0. Available online: http://creativecommons.org/licenses/bynd/4.0/ (accessed on 25 March 2015).

Prusek, T. et al. (2013) 'Deposit models for tube support plate flow blockage in Steam Generators', Nuclear Engineering and Design. Elsevier B.V., 262, pp. 418-428. doi: 10.1016/j.nucengdes.2013.05.017.

Riznic, J. (2017) Steam generators for nuclear power plants, Steam Generators for Nuclear Power Plants. doi: 10.1016/C2015-0-013405.

Sharp, M. (2012) Prognostic Approaches Using Transient Monitoring Methods. Available at: http://trace.tennessee.edu/utk_graddiss/1431/. 